\title{
Inversión máxima para incrementar la disponibilidad de agua en comunidades de la zona limítrofe entre Quintana Roo y Campeche, México
}

\section{Maximum investment to increase water availability in communities in the border area between Quintana Roo and Campeche, Mexico}

DOI: $10.46932 / \mathrm{sfjdv2n5-015}$

Received in: Jun1st, 2021

Accepted in: Sep 30th, 2021

\author{
M.E.S.P. Roberto Mena Rivero \\ TecNM Campus Chetumal \\ E-mail: roberto.mr@chetumal.tecnm.mx \\ Dr. Ricardo Enrique Vega Azamar \\ TecNM Campus Chetumal \\ E-mail: ricardo.va@chetumal.tecnm.mx \\ M.I. Flor Virginia Cruz Gutiérrez \\ IMTA \\ E-mail: flor_cruz@tlaloc.imta.mx \\ M. C. David Gustavo Rejón Parra \\ TecNM Campus Chetumal \\ E-mail: david.rp@chetumal.tecnm.mx \\ Br. Fermín Téllez Gómez \\ TecNM Campus Chetumal \\ E-mail: 115390362@ chetumal.tecnm.mx
}

\section{RESUMEN}

La baja disponibilidad de agua potable de buena calidad puede ser complementada con agua proveniente de la lluvia, si esta última es recolectada en cantidad suficiente para cubrir la demanda, aprovechándose esta combinación para resolver el problema de abastecimiento, todo esto sujeto a restricciones de inversión. El presente trabajo tiene por objetivo presentar un análisis de los sistemas de captación de agua de lluvia existentes en la zona limítrofe entre los estados mexicanos de Quintana Roo y Campeche para estimar los montos de inversión máxima que justifiquen un proyecto de mejora en la disponibilidad de agua de lluvia. En esta investigación se identificó el nivel de aprovechamiento y los requerimientos de los sistemas de captación de agua pluvial en techos (SCAPT) y se determinó el monto de inversión máxima para solucionar esta situación problemática. Los resultados muestran que los SCAPT no se aprovechan debido a limitaciones o subutilización en la superficie de captación y/o capacidad de almacenamiento, por lo que se recurre al suministro de agua en pipas. Se concluye que se puede sustituir el acarreo en pipas por agua de lluvia con un adecuado dimensionamiento de la superficie y el volumen de captación, asegurando el consumo de agua mínimo recomendado, con una inversión menor de la que se requeriría para la implementación de un sistema de abastecimiento de agua convencional.

Palabras clave: inversión máxima en SCAPT, beneficios máximos de SCAPT, análisis de SCAPT, incremento de disponibilidad de agua de lluvia 


\begin{abstract}
Low availability of good quality drinking water can be complemented with rainwater, if the latter is collected in sufficient quantity to cover the demand, taking advantage of this combination to solve the supply problem, all of this subject to investment restrictions. The present work aims to present an analysis of the existing rainwater harvesting (RWH) systems in the border area between the Mexican states of Quintana Roo and Campeche to estimate the maximum investment amount that justifies a project to improve rainwater availability. In this research, the use level and the requirements of rooftop rainwater harvesting (RTRWH) systems were identified and the maximum investment amount needed to solve this problematic situation was determined. Results show that RTRWH systems are not used due to insufficient or underutilized catchment area and / or storage capacity, so water is supplied in tankers. It is concluded that transport in tankers can be replaced by RTRWH with an adequate dimensioning of surface and catchment volume, ensuring the minimum recommended water supply, with a lower investment than that required for the implementation of a conventional water supply system.
\end{abstract}

Keywords: maximum investment in RTRWH, maximum benefits of RTRWH, analysis of RTRWH, increased availability of rainwater.

\title{
1 INTRODUCCIÓN
}

\section{EL AGUA POTABLE Y SU CONSUMO MÍNIMO}

La Organización Mundial de la Salud (OMS) y El Fondo de las Naciones Unidas para la Infancia (UNICEF, por sus siglas en inglés) consideran como agua potable a aquella utilizada para los fines doméstico y la higiene personal, así como para beber y cocinar y aclaran que el agua potable salubre debe cumplir con las características microbianas, químicas y físicas indicadas por la propia OMS o por las autoridades nacionales competentes en temas de calidad del agua potable (CNDH, 2014)

El consumo de agua también está vinculado al nivel de riesgo para la salud, considerándose que un consumo por debajo de 20 1/persona/d es de alto riesgo, 50 1/persona/d de bajo riesgo y por encima de los 100 1/persona/d el riesgo es muy bajo (IMTA, SEMARNAT, \& Río Arronte 2015).

En México, la Comisión Nacional del Agua (CONAGUA) considera al consumo o uso personal como "la aplicación de agua nacional para el uso particular de las personas y del hogar, riego de sus jardines y de árboles de ornato, incluyendo el abrevadero de animales domésticos que no constituya una actividad lucrativa" (CONAGUA, 2004), y que el consumo en las zonas rurales varía dependiendo de distintos factores como el clima y las costumbres locales y recomienda considerar un consumo promedio de 100 1/hab/d. Sin embargo, también sugiere que dependiendo de la magnitud e importancia del proyecto, así como de la información disponible, es recomendable realizar un estudio para determinar los consumos actual y futuro en el área de estudio (CONAGUA, 2015).

\section{Sistemas de Captación de Agua de Lluvia (SCALL)}

Por otra parte, en diversos países, los SCALL han sido utilizados para atender problemas de falta de disponibilidad de agua potable, tal es el caso de Australia, Estados Unidos, Canadá, Colombia, 
Honduras, Brasil y México (Torres, 2019). En Perú, se han propuesto para asegurar hasta $73 \mathrm{~m}^{3}$ de agua por familia al año con precipitaciones cercanas a los $700 \mathrm{~mm}$ anuales en zonas donde se carece de agua potable (Chino-Calli, Velarde-Coaquira, \& Espinoza Calsín, 2016).

Un SCALL está integrado por un conjunto de componentes y accesorios como canaletas, tubos, filtros, separadores de agua, tanques, bombas, y otros que sirven para realizar la recolección, almacenamiento y tratamiento del agua de lluvia. La recolección, tratamiento y almacenamiento para su uso doméstico, conocido como cosecha de lluvia, es una práctica realizada en varias partes del mundo desde la prehistoria (SEDEMA, 2020). Los tipos de SCALL pueden ser variados, y dentro de ellos el sistema de captación de agua pluvial en techos (SCAPT) es el más utilizado para captar o almacenar agua con fines de uso doméstico y/o producción en traspatio (UNAM, 2014). En su diseño se busca almacenar agua de las lluvias y utilizarse durante todo el año.

En México, el Programa Nacional para Captación de Agua de Lluvia y Ecotecnias en Zonas Rurales (PROCAPTAR) tiene por objetivo "impulsar el desarrollo social, el acceso al agua y saneamiento de las viviendas de zonas rurales de mayor marginación, mediante sistemas de captación y almacenamiento de agua de lluvia y tecnologías de tratamiento de aguas residuales a nivel vivienda" (CONAGUA, 2017). Los SCAPT de PROCAPTAR utilizan la superficie del techo de las viviendas (CONAGUA, 2016) y en sus presupuestos consideran los accesorios, canales, filtros, y demás elementos requeridos para conducir el agua a un tanque o captador de agua tipo, es decir, que este tendrá la misma capacidad para todas las viviendas en las que se instale o construya (CONAGUA, 2021).

Los beneficios obtenidos por instalar un SCAPT se dan por al ahorro en costos por acarreo en pipas proyectados en el tiempo y para determinar la rentabilidad en este tipo de proyectos se aplica el valor actual neto (CEPEP, 2011).

\section{La zona limítrofe entre Campeche y Quintana Roo}

El problema de falta de disponibilidad de agua potable y gran cantidad de lluvia se presenta en la zona limítrofe entre Campeche y Quintana Roo, que es una franja territorial en la frontera entre esos dos estados, ubicados al sureste de México (ver Figura 1). Esa zona ha sido motivo de disputas legales desde hace varias décadas (Romero \& Maya, 2017), aun cuando para el Instituto Nacional de Estadística y Geografía (INEGI) ese espacio en conflicto forma parte de Campeche (Alonso, 2020a). 
Figura 1. Zona limítrofe entre Campeche y Quintana Roo.

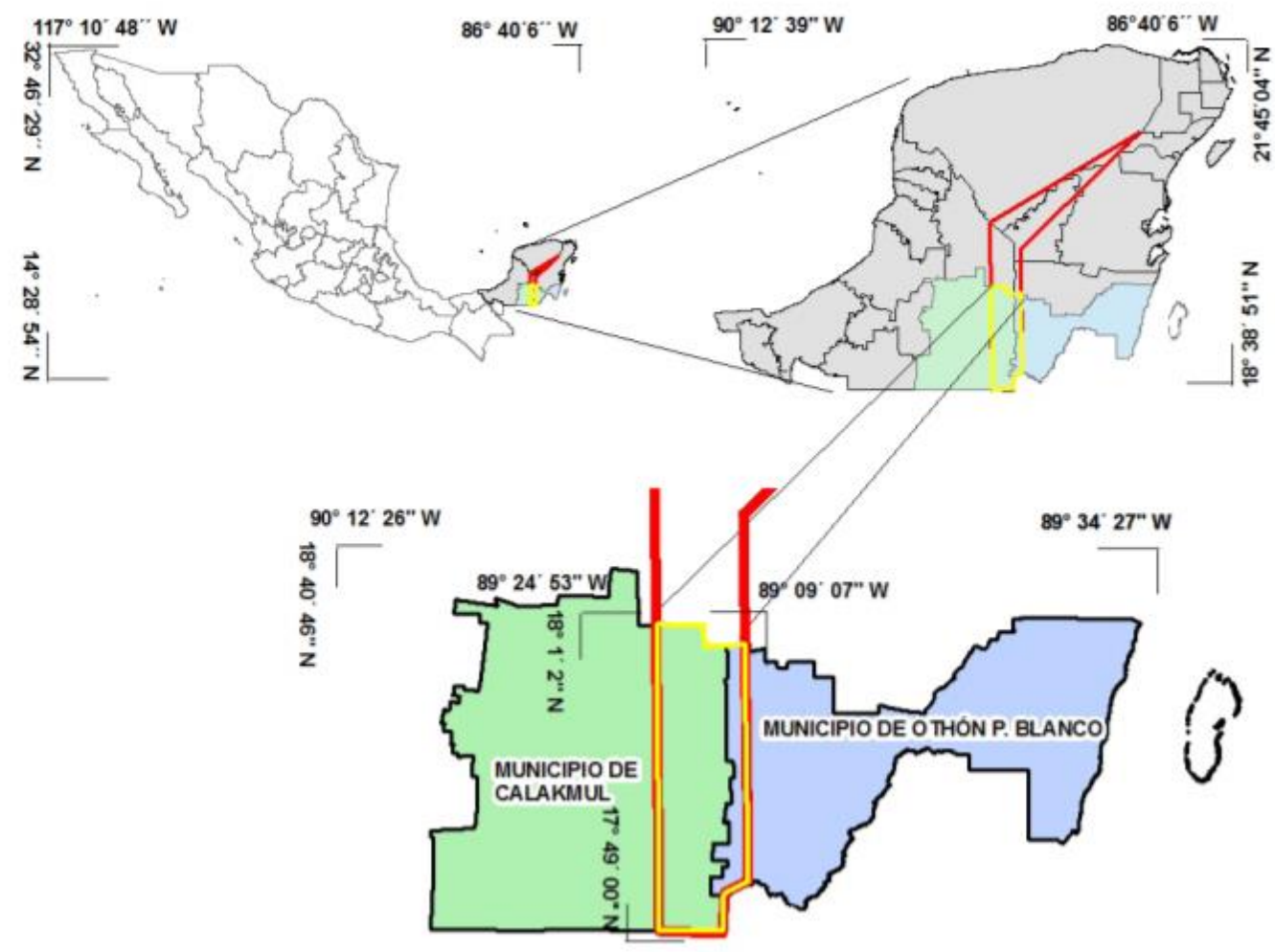

Fuente: Alonso Velasco, I. (2020b)

En esa zona, se han llevado a cabo estudios geohidrológicos en diversas comunidades para identificar agua apta para el consumo humano, pero los resultados indican que el agua no es de buena calidad química, ya que los suelos son de alta permeabilidad lo que hace que presente exceso de sólidos disueltos, en particular yeso, además de que el agua se encuentra a más de 150 metros de profundidad (IMEXSUR, 2011). En contraposición, la precipitación pluvial media en Quintana Roo según el INEGI es de 1300 mm y para Campeche oscila entre 1200 y 2000 mm al año (INEGI, 2021).

En la búsqueda de solución al problema de falta de disponibilidad de agua y aprovechamiento de las precipitaciones pluviales, se han construido jagüeyes y sistemas comunitarios e individuales que captan el agua en los techos, sin embargo, han continuado las quejas de los pobladores por falta de agua, señalando que el agua de lluvia es insuficiente y tienen que solicitar pipas para complementar sus necesidades del vital líquido. El gobierno de Quintana Roo construyó un acueducto para las comunidades de Caobas y San Antonio Soda y con ello puso fin a una solicitud que llevaba varias décadas (Alonso Velasco, I. 2020b). La obra construida beneficiaría a 1,914 habitantes y tuvo un costo de 50 millones 930 mil pesos (GEQROO,2018). Dicha erogación representó un costo per cápita de 26,609 pesos/habitante mientras que en el apartado rural (APARURAL) de las reglas de operación del Programa de Agua Potable, Drenaje y Tratamiento a cargo de la CONAGUA vigente a 2018, publicado en el Diario Oficial de la Federación (DOF) señalaban que la inversión per cápita sería de 10,100 pesos/habitante (DOF: 
30/12/2017, 2017), por lo que la inversión realizada superó en 2.63 veces lo señalado en las reglas vigentes para ese año. En este estudio se presentan los resultados del análisis para la comunidad de "El Sacrificio" en el cual se realizó un diagnóstico de los SCAPT existentes para determinar el nivel de aprovechamiento y la estimación del monto máximo de inversión que justifique la obra de ampliación. Se visitaron 14 comunidades que formaron parte de un convenio de colaboración entre la Comisión de Agua Potable del Estado de Quintana Roo (CAPA) y el Tecnológico Nacional de México (TecNM), Campus Chetumal, denominado "Análisis de los sistemas de abastecimiento de agua potable de las comunidades de Quintana Roo ubicadas en la zona limitrofe con Campeche”.

\section{MATERIALES Y MÉTODOS}

Como ya se mencionó, se realizaron visitas de campo a 14 comunidades de la zona limítrofe, 9 con servicio convencional mediante redes de distribución de agua potable y 5 con abastecimiento mediante SCALL individuales y comunitarios. Las primeras se visitaron para conocer consumo de agua en la zona y las segundas para identificar las características de los SCALL, el consumo de agua proveniente de ellos y la forma cómo se satisface el déficit de agua. Debido a las restricciones de tiempo y presupuesto, durante las visitas se encuestaron 10 viviendas en cada comunidad. A continuación, se presenta el caso de "El Sacrificio".

El Sacrificio tiene 547 habitantes, con una tasa de crecimiento 3.65\%, 113 viviendas y 5.42 habitantes/vivienda, tiene un alto grado de marginación, su localización se puede referir al sur de la localidad de Xpujil, Campeche y al suroeste de Chetumal, Quintana Roo, como puede apreciarse en la Figura 2.

Figura 2. Zona limítrofe entre Campeche y Quintana Roo

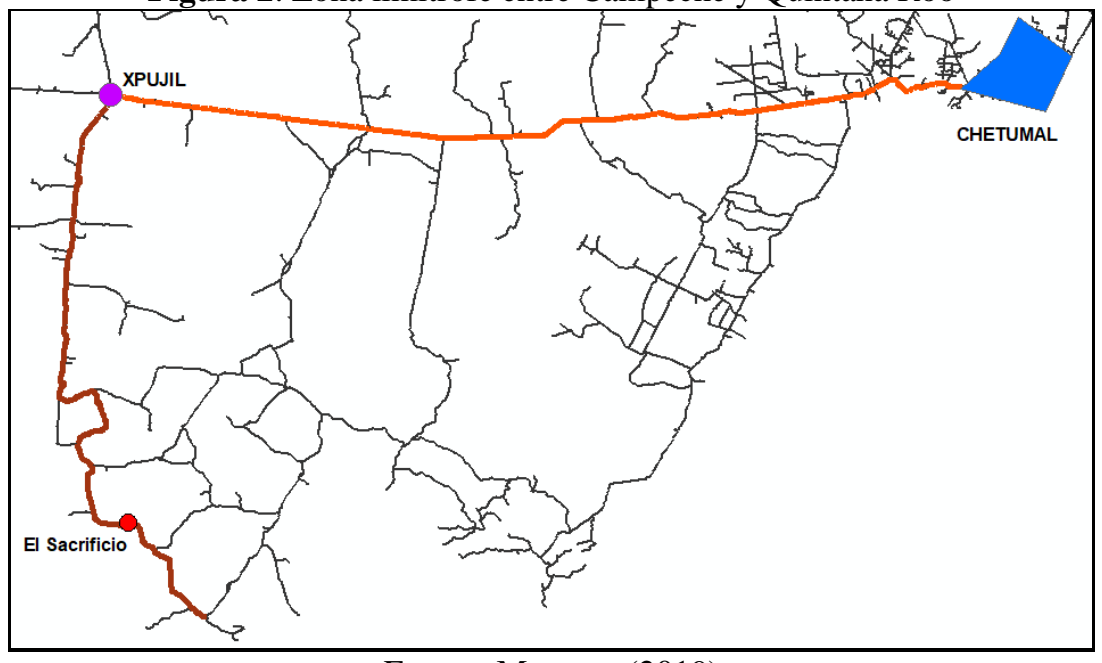

Fuente: Meneses (2019) 
Las viviendas de la comunidad tienen paredes de madera con techo de lámina de zinc y piso de cemento pulido, como se puede ver en la Figura 3.

Figura 3. Casa tipo en la localidad de El Sacrificio.

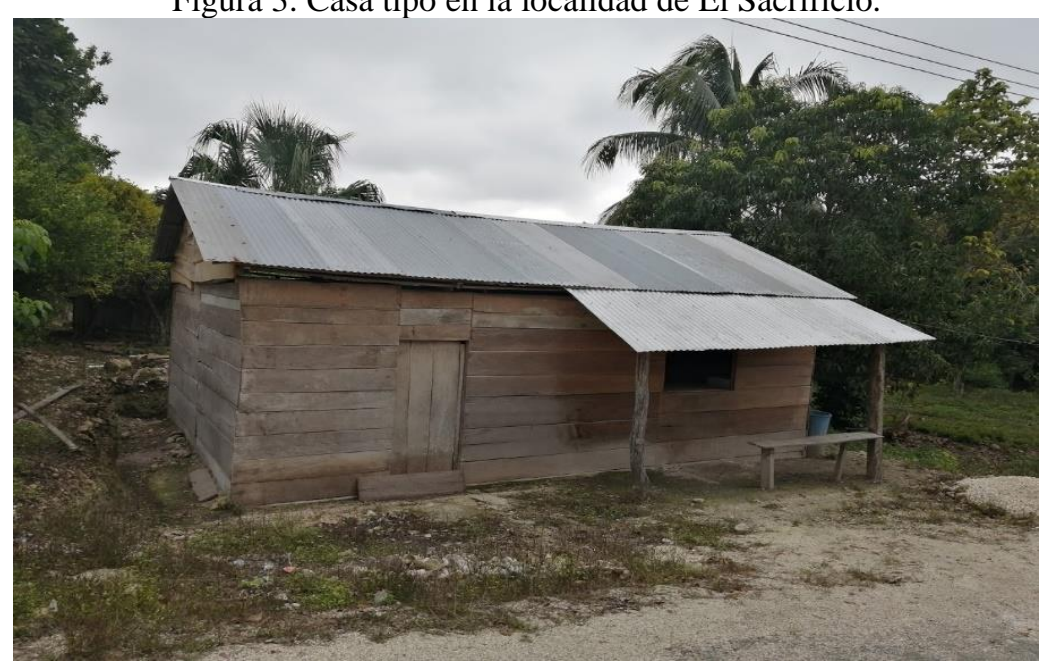

Fuente: Propia (2019)

\subsection{PRECIPITACIONES PLUVIALES}

La precipitación pluvial anual es de 1,352.30 mm al año, presentando valores máximos de 210.88 mm en el mes de junio y mínimo de $35 \mathrm{~mm}$ en febrero y marzo, como se detalla en la tabla 1

Tabla 1. Precipitaciones pluviales promedio en la estación Dos Aguadas

\begin{tabular}{|c|c|}
\hline Mes & Precipitación $(\mathbf{m m})$ \\
\hline Enero & 47.52 \\
\hline Febrero & 35.04 \\
\hline Marzo & 35.50 \\
\hline Abril & 38.66 \\
\hline Mayo & 118.19 \\
\hline Junio & 210.88 \\
\hline Julio & 145.38 \\
\hline Agosto & 140.51 \\
\hline Septiembre & 209.72 \\
\hline Octubre & 199.85 \\
\hline Noviembre & 99.29 \\
\hline Diciembre & 71.74 \\
\hline Total & $\mathbf{1 3 5 2 . 3 0}$ \\
\hline
\end{tabular}

Fuente: Elaboración propia con base en datos de CONAGUA

\subsection{CONSUMO DE AGUA}

Como resultado del trabajo de campo, se identificó mediante encuestas que en la comunidad de El Sacrificio se tiene un consumo de 34 1/hab/d, mientras que en las localidades visitadas que disponen del 
servicio de agua potable tienen un consumo mínimo 33 1/hab/d, máximo de 77 1/hab/d y un promedio ponderado de 49.5 1/hab/d y aun cuando ese consumo está por debajo de los sugeridos por organismos nacionales e internacionales, los habitantes manifestaron que no se han presentado enfermedades de origen hídrico por consumir esa cantidad de agua.

\subsection{DEMANDA DE SCAPT ACTUAL}

1.- Se determinó la demanda de agua mediante la Ecuación 1.

$$
D v=C h * H v * N v
$$

Donde: Dv es la demanda de agua por vivienda, Ch el consumo actual por habitante (1/hab/d), Hv el número de habitantes por vivienda (hab/vivienda). Nv el número de viviendas de la comunidad (viviendas).

2.- Se calculó la superficie de captación mediante la Ecuación 2.

$$
S=\left(\frac{D}{\frac{C e * P P}{1000}}\right)
$$

Donde: $\mathrm{S}$ es la superficie requerida $\left(\mathrm{m}^{2}\right)$, D la demanda ( $\left.\mathrm{m}^{3} / \mathrm{a} n ̃ o\right)$, Pp la precipitación pluvial y Ce el coeficiente de escurrimiento.

3.- Se estimaron las entradas y salidas de agua en el tanque de almacenamiento con ayuda de la Tabla 2.

Tabla 2. Tabla de entrada y salida de agua pluvial del tanque

\begin{tabular}{|c|c|c|c|c|c|}
\hline Mes & Precipitación $(\mathrm{mm})$ & Entrada $\left(\mathrm{m}^{3}\right)$ & Salida $\left(\mathrm{m}^{3}\right)$ & Diferencia & $\begin{array}{c}\text { Diferencia } \\
\text { acumulada } \\
(1)\end{array}$ \\
& $(2)$ & $(3)$ & $(4)$ & $(5)$ & $(6)$ \\
\hline
\end{tabular}

Donde:

Columna 1. Los diferentes meses del año

Columna 2. La precipitación pluvial del mes (mm)

Columna 3. Las entradas se obtuvieron con la Ecuación 3

$$
E=\frac{S * C e * P p}{1000}
$$

Donde: $E$ es la entrada $\left(\mathrm{m}^{3}\right), S$ la superficie de captación, $C e$ el coeficiente de escurrimiento y $P p$ la precipitación pluvial (mm) Columna 4. La demanda es la cantidad de agua demandado por la comunidad

Columna 5. La diferencia entre la entrada y la salida (columna 3 menos columna 4) 
Columna 6. Diferencia acumulada, para el mes 1 su valor es el mismo que la diferencia (columna 5), para el siguiente mes es el valor acumulado del mes 1 más el valor del mes 2 y así sucesivamente.

Por último, el volumen del tanque (Vt) se obtuvo mediante la ecuación (4)

$$
V t=\mid \text { Máximo positivo de columna } 6|+| \text { Máximo negativo de columna } 6 \mid
$$

\subsection{DIAGNÓSTICO DE LOS SCAPT EXISTENTES U OFERTADOS}

Se identificaron las características de los SCAPT existentes tanto individuales como comunitarios, como son superficie de captación y tipo de material, así como el volumen del tanque; posteriormente se estimó el nivel de aprovechamiento de esos elementos y la cantidad de agua de lluvia que se consume actualmente.

Las características de los tanques existentes se obtuvieron mediante visita a la localidad

Para el cálculo de la superficie aprovechada utilizó la Ecuación 5:

$$
S a=\frac{S u}{S i}
$$

Donde: $S a$ es la superficie aprovechada, $S u$ la superficie utilizada y $S i$ la superficie instalada.

La superficie utilizada $S u$ se determinó de dos maneras, la primera considerando el área de captación existente y se siguieron los pasos 2, 3 y 4 señalados para calcular la demanda de un SCAPT y la otra considerando el volumen existente del tanque y determinando la superficie requerida para ello y siguiendo los mismos descritos en este párrafo; eligiendo la menor como la superficie utilizada. Para estimar el nivel aprovechamiento del tanque se utilizó la Ecuación 6.

$$
V a=\frac{V u}{V i}
$$

Donde: $V a$ es el volumen aprovechado, $V u$ el volumen utilizado y $S i$ el volumen instalado.

El volumen utilizado $S u$ se determinó de dos maneras, la primera considerando el área de captación existente y se siguieron los pasos 2, 3 y 4 señalados para calcular la demanda de un SCAPT y la otra considerando el volumen existente del tanque y determinando la superficie requerida para ello y siguiendo los mismos descritos. Se eligió el menor volumen. 


\subsection{COMPARACIÓN OFERTA-DEMANDA DE SCAPT ACTUAL}

Seguidamente se comparó lo existente con lo demandado, para conocer si es suficiente o no la superficie de captación y volumen de tanques existentes. Si la diferencia de volumen entre lo instalado y demandado era negativa se asumió que esa cantidad de agua se satisface mediante pipas.

\subsection{COMPARACIÓN OFERTA-DEMANDA DE SCAPT PARA DIVERSAS CONDICIONES}

Se analizaron 3 escenarios en los cuales se buscó satisfacer un consumo medio de 50 1/hab/d que corresponde a las comunidades que disponen de servicio de agua potable en la zona y está dentro de los limites sugeridos por organismos internacionales. Los escenarios estudiados fueron:

E-1: Considerando las superficies y capacidad de captadores existentes.

E-2: Considerando que se utiliza al $100 \%$ tanto la superficie total de captación y volumen de tanques captadores existentes.

E-3: Considerando la superficie de captación y volumen de tanques captadores requeridos para que se tenga un consumo del $100 \%$ de agua de lluvia.

\subsection{INVERSIÓN MÁXIMA PARA LA AMPLIACIÓN DE SCAPT}

Una vez conocida la cantidad de agua que se consume de la lluvia y la proveniente de pipas, se determinó el número de pipas que entregan agua a la comunidad en un año y éste se multiplicó por el costo del servicio del agua en pipas.

El máximo beneficio al ampliar un SCAPT se consideró como valor presente del ahorro en costos por el servicio de agua en pipas manteniéndolos constantes en el tiempo debido a la migración que existe del campo a las ciudades y considerando como un escenario más probable, para ello se utilizó la Ecuación 7

$$
\operatorname{Imax}=A\left[\frac{(1+i)^{n}-1}{i(1+i)^{n}}\right]
$$

Donde: $I_{m a ́ x}$ es la inversión máxima, $A$ el ahorro en costos de agua en pipas, $i$ la tasa social de descuento para México, $n$ el horizonte de proyecto.

\section{RESULTADOS Y DISCUSIÓN}

\subsection{DEMANDA DE SCAPT EN LA SITUACIÓN ACTUAL}

Para satisfacer la demanda de 34 1/hab/d se requieren captar $9,513 \mathrm{~m}^{3}$ de agua de lluvia al año y para ello debería disponerse de 7,817 $\mathrm{m}^{2}$ de superficie de captación y 2,451 $\mathrm{m}^{3}$ de capacidad en los captadores, los cuales podrían estar divididos en sistemas comunitarios e individuales, como en la actualidad. 


\subsection{DIAGNÓSTICO DE LOS SCAPT EXISTENTES}

En la comunidad existen dos SCAPT comunitarios (ver Figura 4), uno actualmente fuera de servicio y el otro se construyó para aprovechar como área de captación en el domo de la comunidad, pero debido a que el volumen del tanque es insuficiente para almacenar y regular la cantidad de agua de lluvia de entrada y salida, sólo se aprovecha para el consumo de los habitantes el 25\% del agua de lluvia captada, ya que para aprovechar las totalidad de lluvia se requieren $213.21 \mathrm{~m}^{3}$ en el tanque, lo que representa casi 4 veces más el volumen existente. Ver detalles en la Tabla 3.

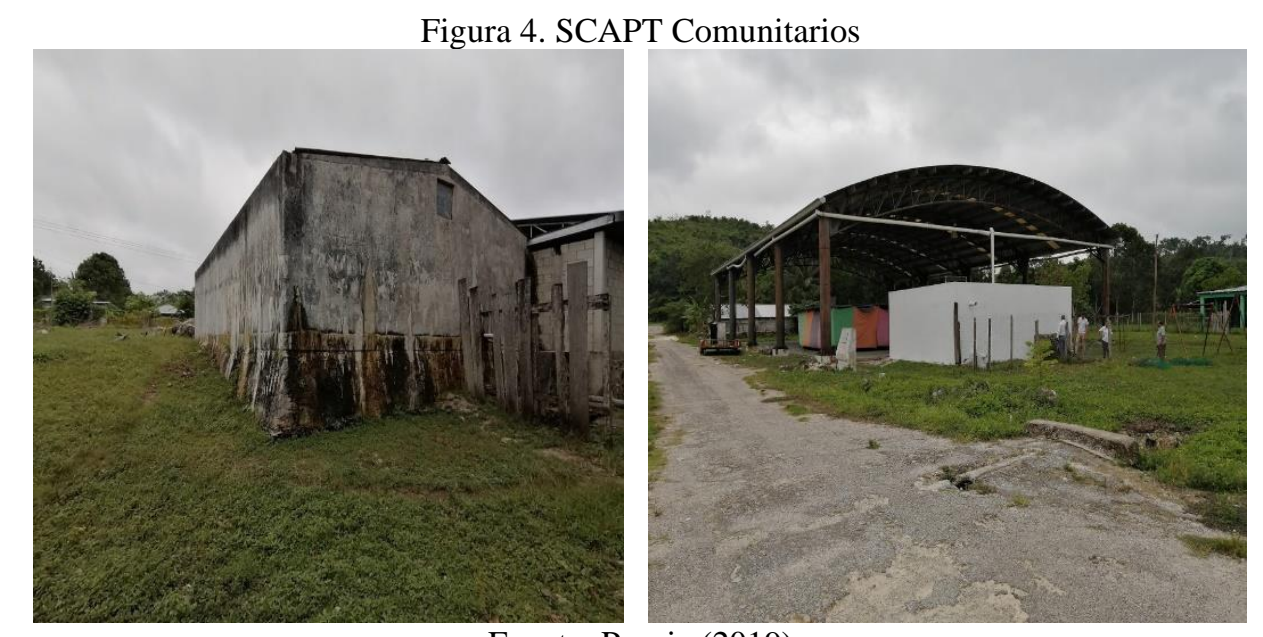

Fuente: Propia (2019)

Tabla 3. Características del SCAPT comunitario en operación

\begin{tabular}{|c|c|c|c|}
\hline Concepto & $\begin{array}{c}\text { Construido o } \\
\text { instalado }\end{array}$ & Aprovechado & $\begin{array}{c}\text { Porcentaje de } \\
\text { aprovechamiento }\end{array}$ \\
\hline Captador 2 Domo & & & $26 \%$ \\
\hline Superficie & $680 \mathrm{~m}^{2}$ & $175 \mathrm{~m}^{2}$ & $26 \%$ \\
\hline Consumo de agua pluvial & $828 \mathrm{~m}^{3} / \mathrm{año}$ & $213 \mathrm{~m}^{3} / \mathrm{año}$ & $72 \%$ \\
\hline Volumen del tanque & $76 \mathrm{~m}^{3}$ & $55 \mathrm{~m}^{3}$ & ${ }^{3}$ \\
\hline
\end{tabular}

Fuente: Elaboración propia

En las viviendas de la comunidad aprovechan la superficie de los techos para captar agua y almacenarla en cisternas pluviales de $13.27 \mathrm{~m}^{3}$ de almacenamiento; sin embargo, debido a que la superficie existente de captación es de $22.50 \mathrm{~m}^{2}$ sólo se aprovechan $27.50 \mathrm{~m}^{3} /$ año de agua de lluvia de $51.51 \mathrm{~m}^{3} /$ año disponible. Para aprovechar toda la cantidad de lluvia que entra y sale por el tanque se requieren $42.32 \mathrm{~m}^{2}$ de superficie (ver Figura 5 y Tabla 4). 
Figura 5. SCAPT Individual

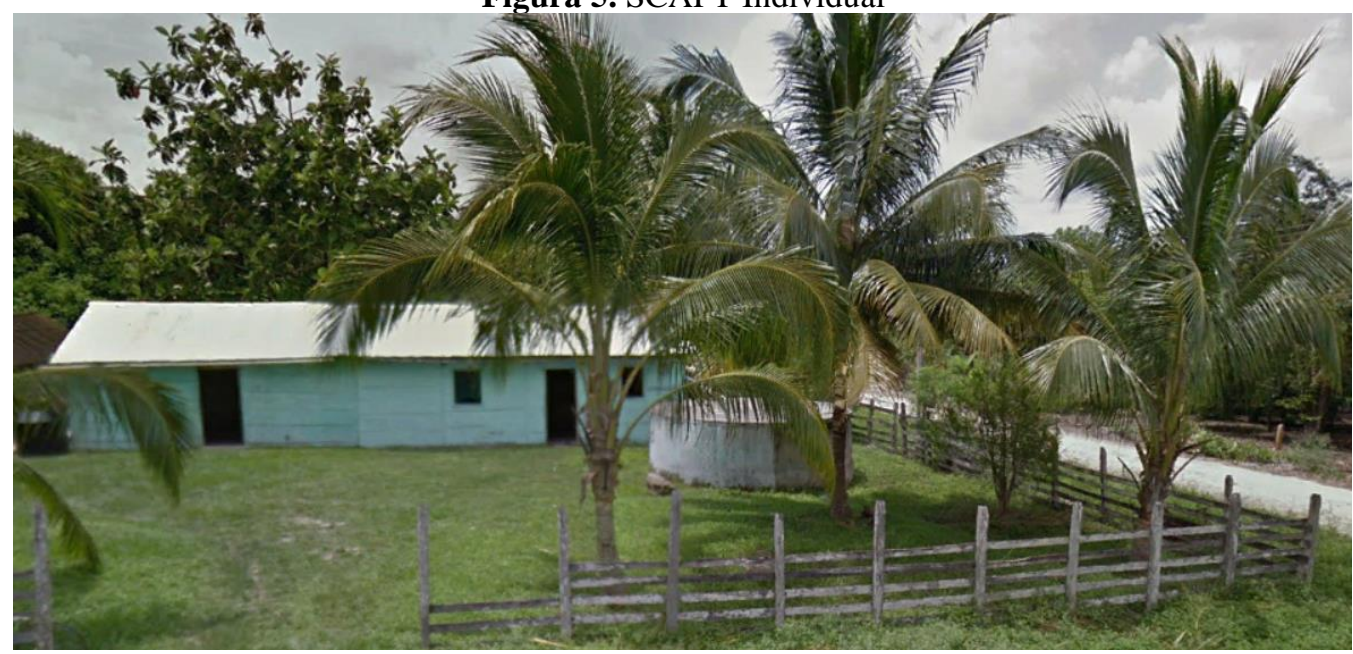

Fuente: Elaboración propia

Tabla 4. Características de los SCAPT individuales

\begin{tabular}{|c|c|c|c|}
\hline Concepto & $\begin{array}{c}\text { Construido o } \\
\text { instalado }\end{array}$ & Aprovechado & $\begin{array}{c}\text { Porcentaje de } \\
\text { aprovechamiento }\end{array}$ \\
\hline $\begin{array}{c}\text { Superficie individual } \\
\text { Consumo individual de agua } \\
\text { pluvial }\end{array}$ & $23 \mathrm{~m}^{2}$ & $23 \mathrm{~m}^{2}$ & $100 \%$ \\
\hline Volumen individual de tanque & $13 \mathrm{~m}^{3} / \mathrm{año}$ & $27 \mathrm{~m}^{3} / \mathrm{año}$ & $53 \%$ \\
\hline \multicolumn{4}{|c|}{ Total } \\
\hline Superficie & $3128 \mathrm{~m}^{2}$ & $3128 \mathrm{~m}^{2}$ & $53 \%$ \\
\hline Consumo de agua pluvial & $7160 \mathrm{~m}^{3} / \mathrm{año}$ & $3806 \mathrm{~m}^{3} / \mathrm{año}$ & $100 \%$ \\
\hline Volumen de tanque & $1845 \mathrm{~m}^{3}$ & $981 \mathrm{~m}^{3}$ & $53 \%$ \\
\hline
\end{tabular}

Fuente: Elaboración propia

Al considerar la superficie total de los SCAPT existentes en la comunidad, se observó un aprovechamiento del $87 \%$, mientras que en el tanque un 54\%, por lo que sólo se logra aprovechar un $50 \%$ del agua de lluvia que se podría captar.

Tabla 5. Total de SCAPT en la comunidad

\begin{tabular}{|c|c|c|c|}
\hline Concepto & $\begin{array}{c}\text { Construido o } \\
\text { instalado }\end{array}$ & Aprovechado & $\begin{array}{c}\text { Porcentaje de } \\
\text { aprovechamiento }\end{array}$ \\
\hline Superficie & 3,808 & 3,303 & $87 \%$ \\
\hline Consumo de agua pluvial & 7,987 & 4,019 & $50 \%$ \\
\hline Volumen de tanque & 1,920 & 1,036 & $54 \%$ \\
\hline
\end{tabular}

Fuente: Elaboración propia

En total, en la comunidad se dispone de $3,303 \mathrm{~m}^{2}$ de superficie de captación y $1,036 \mathrm{~m}^{3}$ de capacidad en los tanques de captación, por lo que sólo se aprovechan 4,019 m³ al año. 


\subsection{ANÁLISIS COMPARATIVO OFERTA-DEMANDA SCAPT EN LA SITUACIÓN ACTUAL}

A partir del comparativo oferta-demanda de SCAPT, se identificó que está instalada la mitad de la superficie de captación y del volumen requerido para aprovechar el agua de lluvia, por lo que sólo se satisface un $42 \%$ del consumo mediante SCAPT (ver Tabla 6).

Tabla 6. Comparativo oferta-demanda de SCAPT

\begin{tabular}{|l|c|}
\hline Demanda de SCAPT & Cantidad \\
\hline Volumen total de agua demandado & $9,513 \mathrm{~m}^{3}$ \\
\hline Superficie demandada & $7,817 \mathrm{~m}^{2}$ \\
\hline Volumen de tanque & $2,451 \mathrm{~m}^{3}$ \\
\hline Oferta total disponible & \\
\hline Volumen total de agua demandado & $4,019 \mathrm{~m}^{3}$ \\
\hline Superficie instalada & $3,303 \mathrm{~m}^{2}$ \\
\hline Volumen de tanque & $1,036 \mathrm{~m}^{3}$ \\
\hline Diferencia de oferta y demanda & \\
\hline Volumen total de agua demandado & $-5,494 \mathrm{~m}^{3}$ \\
\hline Superficie instalada & $-4,514 \mathrm{~m}^{2}$ \\
\hline Volumen de tanque & $-1,415 \mathrm{~m}^{3}$ \\
\hline Consumo & \\
\hline Agua de lluvia & $14 \mathrm{l} / \mathrm{hab} / \mathrm{d}$ \\
\hline Agua de pipas & $20 \mathrm{l} / \mathrm{hab} / \mathrm{d}$ \\
\hline Total & $34 \mathrm{l} / \mathrm{hab} / \mathrm{d}$ \\
\hline
\end{tabular}

Fuente: Elaboración propia

\subsection{ANÁLISIS COMPARATIVO OFERTA-DEMANDA DE SCAPT CON DIFERENTES} CONDICIONES

Para el caso de la implementación de algún proyecto de ampliación de la disponibilidad de agua mediante SCAPT que garantice un consumo de 50 1/hab/d, se tienen los siguientes resultados:

E-1: Con la superficie y capacidad de los captadores existentes, se requerirá de poco más de 8000 $\mathrm{m}^{2}$ de superficie de captación y $2500 \mathrm{~m}^{3}$ de volumen para los captadores, pero se necesitará de casi el doble de agua en pipas de lo que actualmente se suministra.

E-2: Aprovechando al máximo los SCAPT existentes, tanto superficie y como volúmenes máximos, se requerirá adicionalmente cerca de $6000 \mathrm{~m}^{2}$ de superficie de captación y $1500 \mathrm{~m}^{3}$ en volumen de tanques, pero a cambio se reducirá el volumen de agua suministrado por las pipas.

E-3: Si se amplía la superficie de captación existente para que todo el consumo de agua provenga de la lluvia, se requerirá de $8,193 \mathrm{~m}^{2}$ de superficie adicionales y 2,569 $\mathrm{m}^{3}$ de capacidad en los captadores (ver detalles en la Tabla 7). 
Tabla 7. Comparativo oferta-demanda de SCAPT

\begin{tabular}{|c|c|c|c|}
\hline Demanda & E-1 & E-2 & E-3 \\
\hline $\begin{array}{c}\text { Volumen total de agua } \\
\text { demandado }\left(\mathrm{m}^{3} / \mathrm{anno}\right)\end{array}$ & 13,990 & 13,990 & 13,990 \\
\hline Superficie demandada $\left(\mathrm{m}^{2}\right)$ & 11,495 & 11,495 & 11,495 \\
\hline Volumen tanque $\left(\mathrm{m}^{3}\right)$ & 3,604 & 3,604 & 3,604 \\
\hline Oferta & & & 13,990 \\
\hline $\begin{array}{c}\text { Volumen total de agua de lluvia } \\
\text { disponible }\left(\mathrm{m}^{3}\right)\end{array}$ & 4,019 & 7,987 & 11,495 \\
\hline Superficie instalada $\left(\mathrm{m}^{2}\right)$ & 3,303 & 6,563 & 3,604 \\
\hline Volumen de tanque $\left(\mathrm{m}^{3}\right)$ & 1,036 & 2,058 & - \\
\hline Requerimientos & & & - \\
\hline $\begin{array}{c}\text { Volumen total de agua } \\
\text { requerido }\left(\mathrm{m}^{3}\right)\end{array}$ & $-9,971$ & $-6,003$ & - \\
\hline Superficie requerida $\left(\mathrm{m}^{2}\right)$ & $-8,193$ & $-4,932$ & 50 \\
\hline Volumen de tanque $\left(\mathrm{m}^{3}\right)$ & $-2,569$ & $-1,547$ & - \\
\hline Consumo & 14 & & 50 \\
\hline Agua de lluvia & 36 & 29 & 22 \\
\hline Agua de pipas & 50 & 50 & \\
\hline Total & Ful & & \\
\hline
\end{tabular}

Fuente: Elaboración propia

\subsection{INVERSIÓN MÁXIMA PARA LA AMPLIACIÓN DE SCAPT}

La inversión máxima, considerando un ahorro en costos por servicio de 997 pipas/año, un costo promedio de \$1,007 pesos para una pipa de 10,000 litros (PROFECO, 2020), un período de análisis de 20 años y una tasa de descuento del $10 \%$ para pasar de un consumo de 14 1/hab/d de agua de lluvia a 50 1/hab/d, sería de 8.54 millones de pesos, equivalente a 11,181 pesos/habitante, es decir, una inversión per cápita menor a la ejercida en el acueducto para abastecer la comunidad de Caobas y similar a la inversión per cápita actualizada al 2021.

\section{CONCLUSIONES Y RECOMENDACIONES}

Los sistemas de captación de agua de lluvia, que incluyen su recolección, almacenamiento y tratamiento, se han utilizado exitosamente para enfrentar la baja disponibilidad de agua potable en diversos países del mundo. De estos sistemas, el sistema de captación en techos es el más utilizado para aprovechar agua con fines de uso doméstico, lo que, además de representar una excelente alternativa para incrementar la disponibilidad de agua en las comunidades, contribuye a reducir la generación de gases de efecto invernadero por el transporte del agua en pipas.

En el presente trabajo se realizó un análisis en la zona limítrofe entre los estados mexicanos de Quintana Roo y Campeche para estimar los montos de inversión máxima para la mejora en la 
disponibilidad de agua de lluvia, identificándose el nivel de aprovechamiento y los requerimientos de los sistemas de captación en techos.

En el caso de estudio, se determinó que el consumo de agua de lluvia actualmente representa un $42 \%$ del consumo total de agua de la comunidad debido a las limitantes que se tienen en el sistema de captación en techos, ya sea por insuficiencia o subutilización de la superficie de captación y/o del volumen de los tanques. El déficit o subutilización de los tanques captadores individuales puede estar asociado al hecho de que ese tipo de elementos provienen de un proyecto-tipo en el que se utilizan los mismos volúmenes de tanques independientemente de la superficie de captación disponible en las viviendas.

Para asegurar que todo el consumo de agua provenga de la lluvia se requiere ampliar la superficie de captación existente y el volumen de los tanques captadores al triple del existente, lo que conlleva una inversión máxima de \$11,181 pesos/habitante, que es menor de la que se requeriría para la implementación de un sistema convencional de abastecimiento de agua. Esas inversiones deberían ser destinadas a la ampliación de las áreas de captación en techos, así como de los volúmenes de los tanques, hasta unos valores mínimos que garanticen la captación de la cantidad de agua requerida por la comunidad, lo que proporcionaría una mayor cantidad de agua de lluvia con los montos de inversión per-cápita de los programas de apoyo del gobierno federal.

Finalmente, es importante resaltar que el agua de lluvia recolectada en techos puede presentar deficiencias en su calidad, como contaminación microbiológica (patógenos), metales pesados y materia orgánica, aspecto que quedó fuera del alcance de esta investigación. Futuros trabajos deberán considerar los costos asociados a las medidas que se han de considerar para el manejo adecuado de la calidad del agua previo a su recolección y consumo, tales como el diseño, materiales y mantenimiento de los techos de las viviendas, además de la necesidad de algún tipo de tratamiento. 


\section{REFERENCIAS}

Alonso Velasco, I. (2020a). La teoría del conflicto aplicada a los procesos territoriales: el caso de estudio de la Península de Yucatán, México. quivera, 2020-2, 21-41

Alonso Velasco, I. (2020b). Percepción Poblacional De Una Disputa Por Límites Territoriales Entre Las Entidades Federativas Mexicanas De Campeche Y Quintana Roo. Perspectiva Geográfica, 25(2) 123-144. Https://Doi.Org/10.19053/01233769.10463

Caribenoticias. (2012). Quintana Roo Gana Controversia Constitucional Por Conflicto Límitrofe. 03/08/2021, De Caribenoticias Sitio Web: Https://Caribenoticias.Wordpress.Com/2012/07/12/QuintanaRoo-Gana-Controversia-Constitucional-Por-Conflicto-Limitrofe/

Cepep. (2011). Metodología Para La Evaluación Socioeconómica De Proyectos Del Sistema De Captación De Agua Pluvial En Techos.

Chino-Calli, M., Velarde-Coaquira, E., \& Espinoza Calsín, J. J. (2016). Captación De Agua De Lluvia En Cobertura De Viviendas Rurales Para Consumo Humano En La Comunidad De Vilca Maquera, PunoPerú. Revista De Investigaciones Altoandinas - Journal Of High Andean Research, 18(3), 365-373. Https://Doi.Org/10.18271/Ria.2016.226

Clima, Quintana Roo. (2021). Recuperado 12 De Julio De 2021, De INEGI Website: Http://Cuentame.Inegi.Org.Mx/Monografias/Informacion/Qroo/Territorio/Clima.Aspx?Tema=Me CNDH. (2014). Derecho Humano Al Agua Potable Y Saneamiento Basico (P. 2014). P. 2014.

CONAGUA. (2015). Manual De Agua Potable, Alcantarillado Y Saneamiento Manual De Agua Potable , Alcantarillado Y Saneamiento Datos Básicos Para Proyectos De Agua Potable Y Alcantarillado Comisión Nacional Del Agua. México.

CONAGUA. (2016). Lineamientos Técnicos: Sistema De Captación De Agua De Lluvia Con Fines De Abasto De Agua Potable A Nivel Vivienda. Programa Nacional Para Captación De Agua De Lluvia Y Ecotecnias En Zonas Rurales (PROCAPTAR), P. 42. Recuperado De Https://Www.Gob.Mx/Cms/Uploads/Attachment/File/152776/LINEAMIENTOS_CAPTACI_N_PLUV IAL.Pdf

CONAGUA. (2017). Programa Nacional Para Captación De Agua De Lluvia Y Ecotecnias En Zonas Rurales (PROCAPTAR). 04/08/2021, De CONAGUA Sitio Web: Https://Www.Gob.Mx/Conagua/Acciones-Y-Programas/Programa-Nacional-Para-Captacion-De-AguaDe-Lluvia-Y-Ecotecnias-En-Zonas-Rurales-Procaptar

CONAGUA. (2021). Proyectos Tipo Y Presupuestos De Sistemas De Captación De Lluvia (SCALL) Y Saneamiento Básico A Nivel Viviendas En Zonas Rurales.

(DOF: 30/12/2017, 2017) Diario Oficial De La Federación, Publicado El 30 De Diciembre De 2017

GEQROO (2018). Al Fin, Agua Potable En Caobas Y San Antonio Soda: Carlos Joaquín. 02/03/2021, De Gobierno Del Estado De Quintana Roo Sitio Web: Http://Cgc.Qroo.Gob.Mx/Al-Fin-Agua-Potable-EnCaobas-Y-San-Antonio-Soda-Carlos-Joaquin/

PROFECO. (2020). QUIÉN ES QUIÉN EN LOS PRECIOS. 
SEDEMA. (2020). Cosechar La Lluvia, Manual Para Instalarlo En Tu Vivienda. Recuperado De Www.Sedema.Cdmx.Gob.Mx

TECNM (2021). Análisis de los sistemas de abastecimiento de agua potable de las comunidades de Quintana Roo ubicadas en la zona limítrofe con Campeche

Torres Hugues, R (2019) La Captación Del Agua De Lluvia Como Solución En El Pasado Y El Presente. Riha [Online]. 2019, Vol.40, N.2, Pp.125-139. ISSN 1680-0338.

UNAM. (2014). Sistemas De Captación De Agua De Lluvia. Impluvium, I(No. 1), 41. 